\title{
Non-positive curvature and complexity for finitely presented groups
}

\author{
Martin R Bridson*
}

\begin{abstract}
A universe of finitely presented groups is sketched and explained, leading to a discussion of the fundamental role that manifestations of non-positive curvature play in group theory. The geometry of the word problem and associated filling invariants are discussed. The subgroup structure of direct products of hyperbolic groups is analysed and a process for encoding diverse phenomena into finitely presented subdirect products is explained. Such an encoding is used to solve problems of Grothendieck concerning profinite completions and representations of groups. In each context, explicit groups are crafted to solve problems of a geometric nature.
\end{abstract}

Mathematics Subject Classification (2000). Primary 20F65; Secondary 20F67.

Keywords. Geometric group theory, finitely presented groups, non-positive curvature, Dehn functions, filling invariants, decision problems.

\section{Introduction}

When viewed through the eyes of a topologist, a finite group-presentation $\Gamma=$ $\langle\mathcal{A} \mid \mathcal{R}\rangle$ is a concise description of a compact, connected, 2-dimensional $\mathrm{CW}$ complex $K$ with one vertex: the generators $a \in \mathcal{A}$ index the (oriented) 1-cells and the defining relations $r \in \mathcal{R}$ describe the loops along which the boundaries of the 2-cells are attached. $\Gamma$ emerges as the group of deck transformations of the universal cover $\tilde{K}$ and the Cayley graph $\mathcal{C}_{\mathcal{A}}(\Gamma)$ is the 1-skeleton of $\tilde{K}$.

Thus we meet the two main strands of geometric group theory, intertwined as they often are. In the first and most classical strand, one studies actions of groups on metric and topological spaces in order to elucidate the structure of the space and the group. The quality of the insights that one obtains varies with the quality of the action: one may prefer discrete cocompact actions by isometries on spaces with fine geometric structure, but according to context one must vary the conditions on the action, sometimes weakening admission criteria to obtain a more diverse class of groups, sometimes demanding more structure to narrow the focus and study groups and spaces of exceptional character.

This first strand mingles with the second, wherein one regards finitely generated groups as geometric objects in their own right [62], equipped with word metrics:

\footnotetext{
*This work was supported in part by an EPSRC Advanced Fellowship.
} 
given a finite generating set $S$ for a group $\Gamma$ one defines $d_{S}\left(\gamma_{1}, \gamma_{2}\right)$ to be the length of the shortest word in the free group on $S$ that is equal to $\gamma_{1}^{-1} \gamma_{2}$ in $\Gamma$. In other words, $d_{S}$ is the restriction to the vertex set of the standard length metric on the Cayley graph of $\Gamma$. The word metric and Cayley graph depend on the choice of generating set, but their quasi-isometry type does not. Thus one is particularly interested in properties of groups and spaces that are invariant under quasi-isometry. When dealing with such invariants, one is free to replace $\Gamma$ by any space that is quasiisometric to it, such as the universal cover of a closed Riemannian manifold with fundamental group $\Gamma$, where the tools of analysis can be brought to bear.

The techniques of geometric group theory merge into the more combinatorial techniques that held sway in the study of finitely presented groups for most of the twentieth century. At the heart of combinatorial group theory lie the fundamental decision problems first articulated by Max Dehn [50] - the word, conjugacy and isomorphism problems. These continue to play an important role in geometric group theory and provide a unifying theme for the ideas presented here, serving as fundamental measures of the complexity of groups.

In this article and the accompanying lecture I shall discuss two topics that account for much of my work: manifestations of non-positive curvature in group theory, and the geometry of the word problem and associated filling invariants. I shall also explore the subgroup structure of direct products of hyperbolic groups. It transpires that a huge range of phenomena can be encoded into the finitely presented subgroups of such direct products. Such an encoding was used by Fritz Grunewald and I to solve problems of Grothendieck concerning profinite completions and representations of groups; this is explained in section 6 . Throughout the discussion, the reader will find that a prominent role is played by explicit groups crafted to solve problems of a geometric nature.

Acknowledgements. It is a pleasure to thank my coauthors, past and present, for their ideas and companionship. It is also a pleasure to acknowledge the great intellectual debt that I owe to Mikhael Gromov.

\section{The Universe of Finitely Presented Groups}

The picture of the universe of groups that I am about to sketch ${ }^{1}$ is shaded by personal taste and the needs of today but nevertheless I claim that it has intrinsic merit. The point of attempting such a sketch is that it forces one to consider how different classes of groups that arise in disperate settings are related; it challenges one to locate given groups in relation to others and to explain how different classes intersect; and it helps to tease out why certain classes are worthy of particular attention. One asks what theorems hold where, and how various measures of complexity (decision problems, subgroup structure, finiteness properties,...) vary and decay as one moves around the universe. One finds oneself reflecting how problems from elsewhere in mathematics can be encoded in groups of one type but

\footnotetext{
${ }^{1}$ I thank Tim Riley for his skillful rendering of my hand-drawing.
} 
not another, and one starts to wonder how such problems might be transported to a more hospitable region of the universe where one has stricter definitions and better theorems to mount an attack.

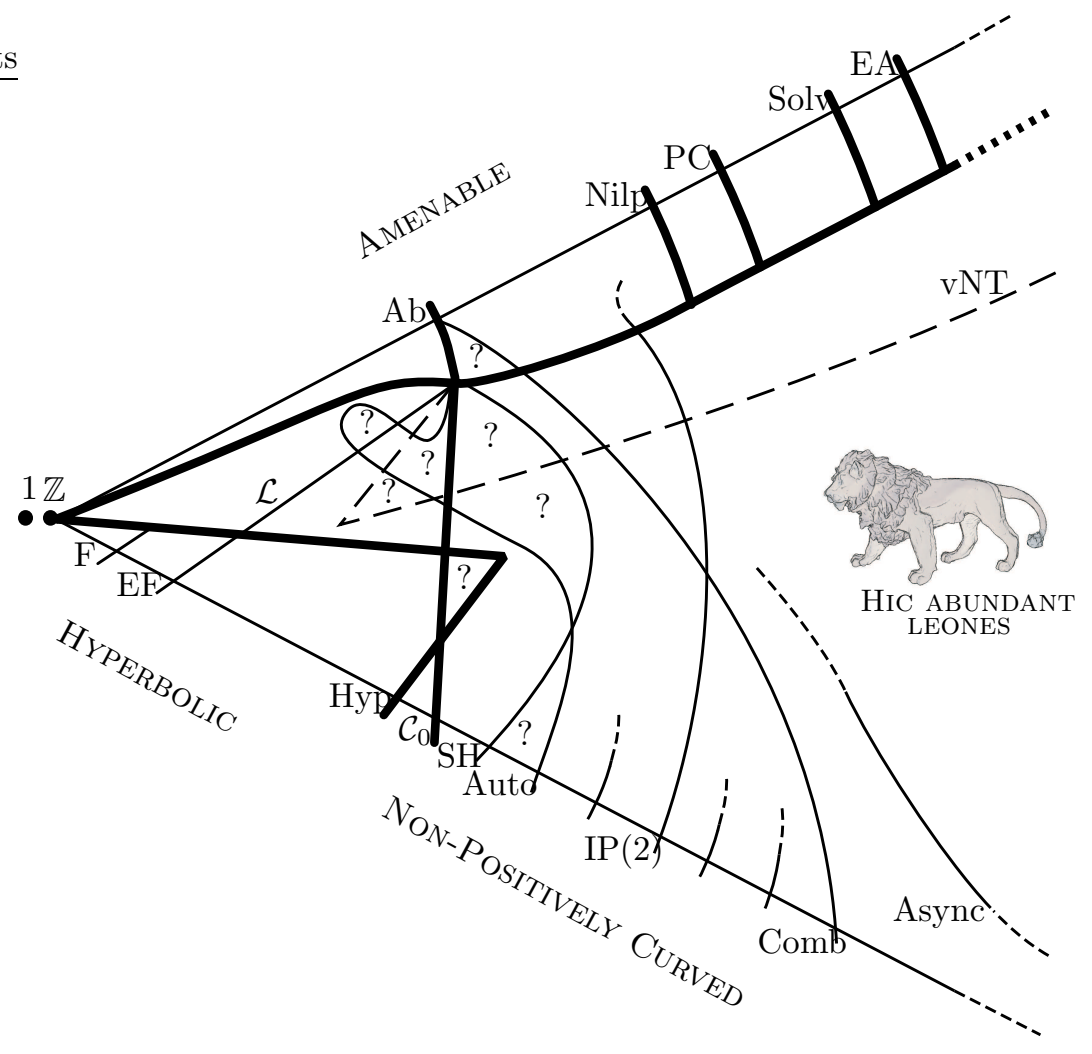

Figure 1. The universe of finitely presented groups.

When approaching group theory from the viewpoint of large-scale geometry, it is natural to blur the distinction between commensurable groups. Thus our universe begins with a single (large and interesting) point labelled 1 representing the finite groups. The simplest infinite group is surely $\mathbb{Z}$, so we have a second point representing the virtually cyclic groups. Here the universe divides. If one wants to retain the safety of commutativity and amenability, one can proceed from $\mathbb{Z}$ to the virtually abelian groups. As one slowly relinquishes commutativity and control over growth and constructability, one passes through the progressively larger classes of (virtually-) nilpotent, polycyclic, solvable and elementary amenable groups, which are marked in the region bounded by a thick line enclosing the amenable groups.

Thinking more freely, instead of taking direct products one might proceed from $\mathbb{Z}$ by taking free products, moving into the class $F$ of virtually free ${ }^{2}$ groups, with

${ }^{2} \mathrm{~F}$ contains only one commensurability class besides $\mathbb{Z}$, but is drawn larger for effect 
their tree-like Cayley graphs. As one proceeds away from F, the infinite curvature of tree-ness gives way to the strictly negative curvature of hyperbolic groups Hyp, then the increasingly weak forms of non-positive curvature that define the classes discussed in the sections that follow: $\mathcal{C}_{0}$, the groups that act properly and cocompactly by isometries on $\mathrm{CAT}(0)$ spaces; $\mathrm{SH}$, the semihyperbolic groups of [2]; the automatic groups Auto of [55]; and the combable and asynchronously combable groups Comb and Async. The line marked IP(2) encloses the groups that satisfy a quadratic isoperimetric inequality (4.3). The thickness of the line delimiting $\mathcal{C}_{0}$ expresses the view that these groups deserve particular attention.

The von Neumann-Tits line (vNT) separates the groups that contain nonabelian free groups from those that do not. For each group below the line, one asks whether its finitely generated subgroups satisfy a Tits alternative: if nonamenable, they should contain a non-abelian free group. One also asks what type of amenable subgroups can arise, cf. (2.3).

In contrast to the other amenable groups, virtually abelian groups are indisputably "non-positively curved". Several classes of non-positively curved groups serve as natural envelopes uniting free and free-abelian groups. These include the right-angled Artin groups, cocompact groups of isometries of CAT(0) cube complexes, and limit groups (marked $\mathcal{L}$ ). The case for placing the class $\mathrm{EF} \subset \mathcal{L}$ of elementarily free groups immediately next to $\mathrm{F}$ is discussed in (1.2). The fundamental group $\Sigma_{g}$ of any closed surface of genus $g \geq 2$ is in EF, lending substance to the tradition in combinatorial group theory that, among non-free groups, it is the $\Sigma_{g}$ that resemble free groups most closely (cf. 1.8).

1.1. Accuracy and completeness. Adapting to today's foci, and so as not to crowd the diagram, I have omitted many natural classes of groups, even non-positively curved groups. In particular, I have not subdivided $\mathcal{C}_{0}$ according to subclasses of $\mathrm{CAT}(0)$ spaces such as $\mathrm{CAT}(-1)$ spaces, spaces with isolated flats, or the classes mentioned above in connection with $\mathbb{Z}^{n}$; these all enjoy important additional properties and have a rich hoard of examples.

The hints at subdivision in Comb are intended to suggest the various subclasses defined by varying the degree of control one demands over the geometry and linguistic complexity of the combing, cf. (3.2). A question mark in a region of interesection indicates that it is unknown if that region is empty. The question mark at the amenable end of Comb asks, more generally, which amenable groups are combable. Likewise, the vague manner in which the boundary of $\operatorname{IP}(2)$ ends reflects a lack of knowledge about which amenable groups have quadratic Dehn functions (4.3). The extent of Async is also unknown, though we know it contains many solvable groups and the fundamental groups of compact 3-manifolds (3.5).

1.2. Limits and ultralimits. From a geometric perspective (as well as others) the virtually nilpotent groups have an indisputable claim to the ground next to abelian groups: they are exactly the groups of polynomial growth, as Gromov proved in the landmark paper [63]. A key idea in that paper is to construct a space on which a group $\Gamma$ of polynomial growth acts by taking the limit in the Gromov- 
Hausdorff topology of a subsequence of the pointed metric spaces $X_{n}=\left(\Gamma, \frac{1}{n} d\right)$, where $d$ is a fixed word metric on $\Gamma$ (the identity serves as a basepoint).

To circumvent the failure of the sequence $X_{n}=\left(\Gamma, \frac{1}{n} d\right)$ to converge in the non-nilpotent case, one fixes a non-principal ultrafilter and takes an ultralimit to obtain an asymptotic cone Cone $\omega_{\omega} \Gamma$. Such cones have been intensively studied in recent years, particularly in connection with quasi-isometric rigidity, e.g. [73]. Their geometry and algebraic topology encode a good deal of information about $\Gamma$ (see $[64,83,54])$. If $\Gamma$ is a non-abelian free group, then $\mathrm{Cone}_{\omega} \Gamma$ is an everywherebranching $\mathbb{R}$-tree (regardless of the choice of $\omega$ ). The class of finitely generated groups that share this property consists precisely of the non-elementary hyperbolic groups. This is just one of the ways in which hyperbolic groups appear as the most commanding generalisation of a free group.

1.3. Next to the free groups: limit groups. Continuing with the idea of taking limits, one might ask which finitely generated groups arise as GromovHausdorff limits of free groups [47]. More precisely ${ }^{3}$, given $\Gamma$, one asks if there exists a finite generating set $S$ for $\Gamma$ and a sequence of finite generating sets $S_{n}$ for a fixed free group $F$ with bijections $S_{n} \rightarrow S$ making the ball of radius $\rho \in \mathbb{N}$ about the identity in the Cayley graph $\mathcal{C}_{S_{n}}(F)$ isomorphic (as a marked graph) to that in $\mathcal{C}_{S}(\Gamma)$ whenever $n \geq N(\rho)$.

The groups that arise in this way are the limit limit groups $\mathcal{L}$, which are nonpositively curved in every reasonable sense [1]. The fundamental importance of this class has been greatly illuminated in recent years by the work of Zlil Sela and others (see subsection 5.3). A fascinating aspect of their study is that the same class of groups emerges from a range of different definitions that make precise the idea of an approximately free group. In terms of first order logic, $\mathcal{L}$ consists of those finitely generated groups that have the same existential theory as a free group [82], while $\mathrm{EF} \subset \mathcal{L}$ consists of those that have the same elementary theory.

1.4. Beware of the lions: encoding. Constructions such as the Higman Embedding Theorem [68] show that one can encode the workings of an arbitrary Turing machine into a finite group-presentation. The groups that one obtains from such constructions will typically not belong to any of our marked classes but rather will lie in the region where the fierce lion is shown defying the groups that submit to the control of our definitions. One should therefore regard the lion as a warning that it is reckless to base a conjecture about arbitrary finitely presented groups on evidence gained along the coasts of the universe.

1.5. Embracing the lions: subdirect products. It is not satisfactory to content oneself with the study of groups in our marked classes alone. In particular one wants to attack problems from elsewhere in mathematics by exploiting the ability to encode arbitrary recursive phenomena into finite grouppresentations. In order to do so without leaving the safety of the regions where

\footnotetext{
${ }^{3}$ The corresponding topology on marked groups is often called the Grigorchuk topology [60].
} 
one has definitions and theorems, one has to find a way of encoding arbitrary presentations into the structure of groups in the labelled classes (the closer to the origin 1 the better). Such encodings exist in remarkable generality, provided one is prepared to accept passing to finitely presented subgroups in the given class. This is the theme of section 5 below, where I explain and exploit the fact that finitely presented subgroups of direct products of hyperbolic groups (but not limit groups) can be made extremely complicated. Section 6 shows how such an encoding can be used to solve problems of interest elsewhere in mathematics.

1.6. Special groups. One might begin an open-minded search for groups of special interest by asking what sort of actions are admitted by an arbitrary finitely presented group. As one tries to improve the quality of the space or action, obstructions emerge and special groups are singled out. For example, when one knows that every finitely presented group is the fundamental group of a closed symplectic 4-manifold and a closed complex manifold, it is natural to ask which groups are fundamental groups of compact Kähler manifolds, or of 3-manifolds, and to pay special attention to such groups, classifying them if possible. It is also stimulating to try to locate them in our map of the universe.

1.7. Classifying spaces. Other special classes emerge as one tries to improve on the construction of $K=K(\mathcal{A}, \mathcal{R})$ in the opening paragraph, making it more highly-connected and looking for a classifying space. Higher finiteness properties emerge and conditions that ensure the existence of a compact $K(\Gamma, 1)$ come into focus, such as the existence of a 1-relator or small-cancellation presentation. Complete non-positively curved spaces serve as classifying spaces but these are not always of the minimum possible dimension [25], [18]. This discrepancy fits into a large body of work in which different notions of dimension measure the cost of choosing between algebraic, topological and geometric models for a group.

Of the classes in figure 1 , hyperbolic, polycyclic and $\mathcal{C}_{0}$ groups all act properly and cocompactly on contractible, finite-dimensional complexes but certain groups in Solv, IP(2) and Async do not. Members of the classes other than Solv, IP(2) and EA have classifying spaces with only finitely many cells in each dimension.

1.8. The first groups and their automorphisms. The view that $\mathbb{Z}^{n}, F_{n}$ and $\Sigma_{g}$ are the most basic of infinite groups begins a rich vein of ideas concerning the automorphisms of these groups. At the level of individual automorphisms, classical facts about integer matrices are paralleled by the NielsenThurston theory of surface automorphisms and, for free groups, the train-track technology of Bestvina, Feighn and Handel [10], [11].

The analogies between the outer automorphism groups $\operatorname{GL}(n, \mathbb{Z}), \operatorname{Out}\left(F_{n}\right)$ and $\operatorname{Mod}_{g} \cong \operatorname{Out}\left(\Sigma_{g}\right)$ (the mapping class group) go far beyond the observation that $\operatorname{GL}(2, \mathbb{Z}) \cong \operatorname{Out}\left(F_{2}\right) \cong \operatorname{Mod}_{1}$. Indeed much of the work on mapping class groups and automorphisms of free groups is premised on such analogies [9, 91, 44]. Karen Vogtmann has been particularly influencial in promoting this idea. 


\section{CAT(0) spaces and their isometries}

The theory of CAT(0) spaces has had a huge impact in recent years, not only in geometric group theory but also in the study of low-dimensional manifolds and rigidity phenomena in geometry. (This influence, which owes much to the work of Gromov, is easy to discern in the proceedings of recent ICMs including this one.) My purpose here is not to survey this field but rather to highlight some features of the basic theory with an eye on their quasi-fication in the next section. My book with André Haefliger [35] provides a thorough introduction to the subject.

2.1. CAT(0) spaces. Following A.D. Alexandrov, one defines non-positive curvature in the context of length spaces $X$ by means of the $C A T(0)$ inequality, which requires that small triangles in $X$ be no fatter than their comparison triangles in the Euclidean plane. Thus one compares triangles $\Delta=\Delta\left(x_{1}, x_{2}, x_{3}\right)$ consisting of three points $x_{1}, x_{2}, x_{3} \in X$ and three geodesic segments $\left[x_{i}, x_{j}\right]$ to triangles $\bar{\Delta}\left(\bar{x}_{1}, \bar{x}_{2}, \bar{x}_{3}\right) \subset \mathbb{E}^{2}$ with $d\left(\bar{x}_{i}, \bar{x}_{j}\right)=d\left(x_{i}, x_{j}\right)$. A point $\bar{p}$ on the line segment $\left[\bar{x}_{i}, \bar{x}_{j}\right]$ is called a comparison point for $p \in\left[x_{i}, x_{j}\right]$ if $d\left(x_{i}, p\right)=d\left(\bar{x}_{i}, \bar{p}\right)$.

A geodesic space is said to be a $C A T(0)$ space if for all triangles $\Delta$ in that space, $d(p, q) \leq d(\bar{p}, \bar{q})$ for all comparison points $\bar{p}, \bar{q} \in \bar{\Delta}$. And a metric space $X$ is defined to be non-positively curved if every point of $X$ has a neighbourhood that, when equipped with the induced metric, is a CAT(0) space. Similarly, one defines the notion of a CAT $(-1)$ space by taking comparison triangles in the hyperbolic plane, and one defines a space to be negatively curved (in the sense of A.D. Alexandrov) if it is locally $\operatorname{CAT}(-1)$.

Because the CAT(0) condition encapsulates the essence of non-positive curvature so well, non-positively curved metric spaces satisfy many of the elegant features inherent in the theory of Riemannian manifolds of non-positive sectional curvature. At the heart of the theory lie local-to-global phenomena that spring from the fact that the metric on a $\operatorname{CAT}(0)$ space $X$ is convex: if $c_{1}, c_{2}:[0,1] \rightarrow X$ are geodesics, then for all $t \in[0,1]$

$$
d\left(c_{1}(t), c_{2}(t)\right) \leq(1-t) d\left(c_{1}(0), c_{2}(0)\right)+t d\left(c_{1}(1), c_{2}(1)\right) .
$$

This inequality implies that there is a unique geodesic segment joining each pair of points in $X$ and that $X$ is contractible. The most important example of a local-to-global phenomenon is the Cartan-Hadamard Theorem: If a complete, simply-connected metric space is non-positively curved, then it is $\mathrm{CAT}(0)$ space. (See Chapter II.4 of [35] for a more general result and references.)

It follows from this theorem that compact non-positively curved spaces have contractible universal covers and hence provide classifying spaces. The usefulness of this observation is greatly enhanced by two facts. First, Gromov's link condition ([35] p.206) enables one to reduce the question of whether a polyhedral complex supports a metric of non-positive curvature to a question about the geometry of links in that complex; this allows arguments that proceed by induction on the dimension of the complex, and if the cells are sufficiently regular (e.g. cubes) it can lead to purely combinatorial criteria for the existence of metrics of non-positive 
curvature. Secondly, gluing theorems ([35] II.11) allow one to preserve non-positive curvature while combining spaces according to group-theoretic constructions that one wishes to perform at the level of $\pi_{1}$, such as amalgamated free products $\Gamma_{1} *_{\mathbb{Z}} \Gamma_{2}$.

2.2. Splitting theorems. A rich vein of ideas begins with Alexandrov's observation that, when considering a triangle $\Delta$ in a complete $\operatorname{CAT}(0)$ space $X$, if one gets any non-trivial equality in the $\operatorname{CAT}(0)$ inequality, then $\Delta$ spans an isometrically embedded triangular Euclidean disc in $X$. This observation leads one quickly to the fact that any pair of geodesic lines $\mathbb{R} \rightarrow X$ a bounded distance apart must bound a flat strip in $X$, and thence to a Product Decomposition Theorem: Let $c: \mathbb{R} \rightarrow X$ be a geodesic line and let $P$ be the set of geodesic lines $c^{\prime}$ contained in a bounded neighborhood of $c(\mathbb{R})$. Let $c_{0}^{\prime} \in c^{\prime}(\mathbb{R})$ be the unique point closest to $c(0)$ and let $X_{c}^{0}=\left\{c_{0}^{\prime} \mid c^{\prime} \in P\right\}$. Then $\bigcup\left\{c^{\prime}(\mathbb{R}) \mid c^{\prime} \in P\right\}$ is isometric to $X_{c}^{0} \times \mathbb{R}$.

This places severe restrictions on the way groups can act on CAT(0) spaces.

Proposition 2.1. If $\Gamma$ acts properly and cocompactly by isometries on a $\mathrm{CAT}(0)$ space and $\gamma \in \Gamma$ has infinite order, then the centralizer $C_{\Gamma}(\gamma)$ has a subgroup of finite index that splits as a direct product $C_{0}=N \times\langle\gamma\rangle$.

To prove this, one considers the union $\operatorname{Min}(\gamma)$ of the geodesic lines in $X$ that are left invariant by $\gamma$. The Product Decomposition Theorem gives a splitting $\operatorname{Min}(\gamma)=\mathrm{Y}_{0} \times \mathbb{R}$. The centralizer $C_{\Gamma}(\gamma)$ preserves $\operatorname{Min}(\gamma)$ and its splitting, acting by translations on the second factor. $C_{\Gamma}(\gamma)$ is finitely generated (3.3), so the image of $C_{\Gamma}(\gamma) \rightarrow \operatorname{Isom}(\mathbb{R})$ is isomorphic to $\mathbb{Z}^{r}$ for some $r$. Projecting onto a direct factor of $\mathbb{Z}^{r}$ gives an epimorphism $\phi$ from a subgroup of finite index $C_{0} \subset C_{\Gamma}(\gamma)$ to $\langle\gamma\rangle$. Hence $C_{0}=\operatorname{ker} \phi \times\langle\gamma\rangle$. A similar argument proves:

If a finitely generated group $\Gamma$ acts by isometries on a $\mathrm{CAT}(0)$ space (the action need not be proper) and a central subgroup $A \cong \mathbb{Z}^{n}$ acts freely by hyperbolic isometries, then $\Gamma$ has a subgroup of finite index that contains $A$ as a direct factor.

These results give us our first glimpse of the fact that centralizers play an important role in non-positively groups. Many groups that lie in $\mathrm{SH}$ and Auto are seen not to lie in $\mathcal{C}_{0}$ because their centralizers do not virtually split; examples include mapping class groups [77] and central extensions of hyperbolic groups [79]. Such central extensions are defined by bounded cocycles and hence are quasiisometric to the corresponding direct products. It follows that $\mathcal{C}_{0}$ is not closed under quasi-isometry.

More elaborate arguments akin to the one sketched above allow one to generalize splitting theorems proved in the Riemannian setting by D. Gromoll and J. Wolf [61] and B. Lawson and S. Yau [74] (see [35] pp. 239-253).

Theorem 2.2. If a group $\Gamma=\Gamma_{1} \times \Gamma_{2}$ with trivial centre acts properly and cocompactly by isometries on a CAT(0) space in which geodesics can be extended locally, then $\Gamma_{1}$ and $\Gamma_{2}$ also admit such actions.

Theorem 2.3. If $\Gamma$ acts properly and cocompactly by isometries on a $\mathrm{CAT}(0)$ space $X$, then every solvable subgroup $S \subset \Gamma$ is finitely generated and virtually abelian. Moreover, $S$ leaves invariant an isometrically embedded copy of Euclidean space $\mathbb{E}^{n} \hookrightarrow X$ on which is acts cocompactly. 
A refinement of the last part of this theorem can be used to identify constraints on the length functions $\gamma \mapsto \min _{x} d(x, \gamma \cdot x)$ associated to semisimple actions on CAT(0) spaces. Such constraints serve as obstructions to the existence of actions both absolutely and in certain dimensions [18], [25], [57].

2.3. Subgroups in $\mathcal{C}_{\mathbf{0}}$. As one pursues an understanding of the groups that act properly and cocompactly by isometries on $\mathrm{CAT}(0)$ spaces one finds increasingly subtle obstructions to the existence of metrics of non-positive curvature on aspherical spaces. In order to get at the heart of this subtlety one wants to bypass the obstructions to semisimple actions. One way of doing this is to focus on the finitely presented subgroups of fundamental groups of compact non-positively curved spaces. It transpires that such subgroups form a much more diverse class than the fundamental groups themselves — see [35] III.Г.5, [29] and Section 5.

In very low dimensions, finitely presented subgroups are well-behaved [29]. If $\Gamma$ is the fundamental group of a compact non-positively curved manifold of dimension $\leq 3$ (allowing boundary) then so too is each of its finitely generated subgroups.

A similar result holds for complexes of dimension $\leq 2$, except that one has to impose the hypothesis that the subgroups are finitely presented. In higher dimensions all manner of additional obstructions emerge: higher finiteness conditions, the complexity of decision problems, the structure of centralizers, etc. The subtlety of the situation is illustrated by the following construction [29].

Theorem 2.4. There exist pairs of closed aspherical manifolds $N^{n} \hookrightarrow M^{n+1}$ with the following properties: $M$ supports a metric of non-positive sectional curvature; $\pi_{1} N \hookrightarrow \pi_{1} M$ is a quasi-isometric embedding; the centralizers of all finite subsets in $\pi_{1} N$ are fundamental groups of closed aspherical manifolds and have solvable word and conjugacy problems; but $\pi_{1} N$ is not semihyperbolic, and hence $N$ does not support a metric of non-positive curvature.

One sees that $\pi_{1} N$ is not semihyperbolic by examining the complexity of the word problem in centralizers: although $\pi_{1} N$ satisfies a polynomial isoperimetric inequality, the centralizers of certain elements do not.

2.4. Complexes of Group. An important instance of the local-to-global effect of non-positive curvature is the Developability Theorem for non-positively curved complexes of groups. This was inspired by Gromov and proved by Haefliger, who placed it in the more general setting of groupoids of local isometries [35] p.584.

Complexes of groups were introduced by Haefliger to describe groups actions on 1-connected polyhedral complexes in terms of suitable local data on the quotient. If a complex of groups arises from such an action then it is said to be developable. In contrast to the 1-dimensional situation (graphs of groups), complexes of groups are not developable in general. But, crucially, they are if they satisfy a (local) non-positive curvature condition [35]. A full account of the theory is given in the final chapters of our book [35]. In recent years, this theory has played an important role in the construction of group actions. 


\section{Non-Positively Curved Groups}

In this section I'll describe the manifestations of non-positive curvature in group theory that arise from the following strategy. One starts by identifying a robust feature of CAT(0) spaces that encapulates much of their large-scale geometry. Then, given a group $\Gamma$ with generators $\mathcal{A}$ acting properly and cocompactly by isometries on a $\operatorname{CAT}(0)$ space $X$ with basepoint $p$, one tries to articulate what remains of this feature when it is pulled-back to the Cayley graph $\mathcal{C}_{\mathcal{A}}(\Gamma)$ via the $\Gamma$-equivariant quasi-isometry sending the edge $[1, a](a \in \mathcal{A})$ to the geodesic $[p, a . p]$. One wants to define a group to be non-positively curved if it satisfies the resulting condition. The condition should be strong enough to facilitate a range of theorems analogous to what one knows about the prototypical groups of isometries, but one wants to avoid unnecessary hypotheses.

3.1. Hyperbolic groups. Let me recall how such a strategy is implemented in the hyperbolic case. The prototypical hyperbolic group is a group $\Gamma$ that acts properly and cocompactly by isometries on a $\operatorname{CAT}(-1)$ space $X$. By comparing geodesic triangles $\Delta=\Delta(x, y, z)$ in $X$ to triangles $\bar{\Delta} \subset \mathbb{H}^{2}$, one sees that there is a universal constant $\delta$ such that the distance from any point $q \in[x, y]$ to $[x, z] \cup[z, y]$ is at most $\delta$. Moreover, quasigeodesics in $\operatorname{CAT}(-1)$ spaces stay uniformly close to geodesics, so $(\lambda, \varepsilon)$-quasigeodesic triangles in $X$ are uniformly thin in the same sense (with a different $\delta$ ). The quasi-isometry $\mathcal{C}_{\mathcal{A}}(\Gamma) \rightarrow X$ sends geodesic triangles in $\mathcal{C}_{\mathcal{A}}(\Gamma)$ to $(\lambda, \varepsilon)$-quasigeodesic triangles in $X$, where $\lambda$ and $\varepsilon$ depend on $\mathcal{A}$ and $p$. Therefore geodesic triangles in $\mathcal{C}_{\mathcal{A}}(\Gamma)$ are also uniformly thin. One takes this to be the defining property of a hyperbolic group.

Gromov's great insight is that because the thin triangles condition (which has many reformulations [35] p.407) encapsulates so much of the essence of the largescale geometry of CAT $(-1)$ spaces, the groups whose Cayley graphs satisfy this condition share almost all of the properties enjoyed by the groups of isometries that were their prototypes. For example, every hyperbolic group $\Gamma$ acts properly and cocompactly on a contractible cell complex, has only finitely many conjugacy classes of finite subgroups, and contains no copy of $\mathbb{Z}^{2}$. Hyperbolic groups also enjoy a great deal of algorithmic structure. They are precisely the groups with linear Dehn functions. Their conjugacy problems can be solved in less than quadratic time, and conjugacy for finite subsets can also be determined efficiently [36]. Strikingly, the translation lengths $\tau(\gamma)=\lim d\left(1, \gamma^{n}\right) / n$ of elements of infinite order are rational numbers with bounded denominators $[65,51]$. And given a finite generating set $\mathcal{A}$, the set of geodesic words for $\Gamma$ is a regular language, i.e. there is a finite state automaton that recognises which words label geodesics in $\mathcal{C}_{\mathcal{A}}(\Gamma)$.

3.2. The pantheon of non-positively curved groups. The behaviour of geodesics described in (2.1) explains much of the global geometry of CAT(0) spaces, so we apply our strategy to this condition. For the prototype of $\Gamma$ acting on $X$, the geodesics $[p, \gamma \cdot p]$ pull-back to quasi-geodesics $\sigma_{\gamma}$ connecting 1 to $\gamma$ in $\mathcal{C}_{\mathcal{A}}(\Gamma)$. There is no loss of generality in assuming that $\sigma_{\gamma}$ is an edge-path. By identifying $\sigma_{\gamma}$ with the word in the generators $\mathcal{A}^{ \pm 1}$ that labels it, we get a map 


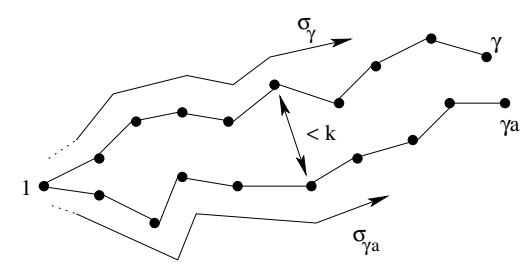

Figure 2. The fellow-traveller property

$\sigma: \gamma \mapsto \sigma_{\gamma}$ to the free monoid $\left(\mathcal{A} \cup \mathcal{A}^{-1}\right)^{*}$, where the image $\sigma(\Gamma)$ can be studied as a formal language. Following Bill Thurston [55], one calls $\sigma$ a combing.

The convexity of the metric on $X(2.1)$ implies that there exists a constant $k>0$ such that for all $\gamma, \gamma^{\prime} \in \Gamma$,

$$
d\left(\sigma_{\gamma}(t), \sigma_{\gamma^{\prime}}(t)\right) \leq k d\left(\gamma, \gamma^{\prime}\right)
$$

for all $t \leq \max \left\{\left|\sigma_{\gamma}\right|,\left|\sigma_{\gamma}\right|\right\}$. This is called the fellow-traveller property. A group that admits a combing (normal form) with this property is said to be combable.

All hyperbolic groups $\Gamma$ are combable: one can take $\sigma_{\gamma}$ to be any geodesic from 1 to $\gamma$ but it is better to be systematic, choosing the first word in the dictionaryordering obtained by ordering the generating set. If one adopts this systematic choice, then $\sigma(\Gamma)$ will be a regular language. With this in mind, ought one to include regularity as part of the definition of a non-positively curved group?

Requiring $\sigma(\Gamma)$ to be regular leads to the theory of automatic groups, which sprang from conversations between Bill Thurston and Jim Cannon on the algorithmic properties of Kleinian groups [46] and grew into a rich theory with large classes of natural examples. It is described in detail in the book by Epstein et al. [55]. Automatic groups lend themselves well to practical computation.

If $\sigma(\Gamma)$ is a regular language, it follows from the fellow-traveller property that the paths $\sigma_{\gamma}$ are quasi-geodesics with uniform constants. But in the absence of regularity it is unclear if one eliminates groups by imposing conditions on the length of $\sigma_{\gamma}$. This question is related to the complexity of the word problem for combable groups: a diagrammatic argument [21] shows that if one has a function $L: \mathbb{N} \rightarrow \mathbb{N}$ bounding the length of combing lines, $\left|\sigma_{\gamma}\right| \leq L(d(1, \gamma))$, then the Dehn function of $\Gamma$ satisfies $\delta_{\Gamma}(n) \preccurlyeq n L(n)$; in particular, if the combing lines are quasi-geodesics then the group satisfies a quadratic isoperimetric inequality.

Another dilemma arises from the observation that although the convexity of $t \mapsto d\left(c_{1}(t), c_{2}(t)\right)$ in the $\mathrm{CAT}(0)$ setting follows easily from the special case $c_{1}(0)=$ $c_{2}(0)$, the analogous statement for groups is false. Thus it is unclear whether every group $\Gamma$ that admits a combing with the fellow-traveller property must admit a combing $\sigma$ with the stronger property

$$
d\left(a . \sigma_{a^{-1} \gamma a^{\prime}}(t), \sigma_{\gamma}(t)\right) \leq k
$$

for all $a, a^{\prime} \in \mathcal{A}$ and $\gamma \in \Gamma$. Groups that admit such combings are said to be bicombable [90]. If, in addition, the combing lines are quasi-geodesics (with uniform 
constants) then, following Alonso-Bridson [2], one says the group is semihyperbolic; this is the smallest of the classes we are discussing that includes $\mathcal{C}_{0}$.

This completes my brief sketch of how the classes Comb, Auto and SH marked in figure 1 present themselves for study. But are these classes distinct? Geometric group theory in the 1990s was marred by the absence of examples to distinguish between them but this situation was now been resolved [26].

Theorem 3.1. There exist combable groups that are neither bicombable nor automatic.

Once one knows that combable groups need not be automatic, it is natural to ask what classes or groups are incorporated if one places weaker constraints on the linguistic complexity of the formal language $\sigma(\Gamma) \subseteq\left(\mathcal{A} \cup \mathcal{A}^{-1}\right)^{*}$. Among full abstract families of languages the regular, context-free and indexed languages form a hierarchy $\operatorname{Reg} \subset \mathrm{CF} \subset$ Ind. If $\sigma(\Gamma)$ lies in a family $\mathcal{F}$, one calls $\sigma$ an $\mathcal{F}$-combing.

Theorem 3.2. [26] There exist Ind-combable groups that are not automatic; some of these have quadratic Dehn functions, others have cubic ones.

A fascinating aspect of the struggle to understand different manifestations of non-positive curvature concerns the free-by-cyclic groups $F_{n} \rtimes \mathbb{Z}$. One feels these groups ought to conform to the expectations of non-positive curvature, yet they remain enigmatic. Since free-group automorphisms are more complicated than surface automorphisms, free-by-cyclic groups are more complicated than the fundamental groups $\Sigma_{g} \times \mathbb{Z}$ of 3-manifolds that fibre over the circle. We know that $F_{n} \rtimes_{\phi} \mathbb{Z}$ need not be automatic [17] but we don't know for which $\phi$ it is; nor when it lies in $\mathcal{C}_{0}$. Daniel Groves and I used the train-track technology of $[10,11]$ to prove that all $F_{n} \rtimes \mathbb{Z}$ lie in $\operatorname{IP}(2)$, but this is highly non-trivial.

3.3. Subgroups. We saw in (2.2) that centralizers of groups in $\mathrm{SH}$ do not virtually split as they do in $\mathcal{C}_{0}$. However, $\mathrm{SH}$ is closed under passage to centralizers of finite subsets. Theorem 2.2 extends to $\mathrm{SH}$. As for Theorem 2.3, one can prove that any polycyclic subgroup $P$ of $\Gamma \in \mathrm{SH}$ must be virtually abelian and that $P \hookrightarrow \Gamma$ must be a quasi-isometric embedding, but it is unknown if abelian subgroups must be finitely generated. The positive part of this statement ultimately derives from the fact that translation numbers $\tau(\gamma)=\lim d\left(1, \gamma^{n}\right) / n$ are positive for elements of infinite order, while the negative part derives from the fact that, unlike in Hyp and $\mathcal{C}_{0}$, one does not know if the set of these numbers is discrete. These results are proved in [2] and [90] using ideas from [59].

In combable groups, the control over centralizers is lost [26].

3.4. The conjugacy and isomorphism problems. If two rectifiable loops $c_{0}, c_{1}$ in a compact, non-positively curved space $X$ are freely homotopic, they are homotopic through loops $c_{t}$ of length $l\left(c_{t}\right) \leq \max \left\{l\left(c_{0}\right), l\left(c_{1}\right)\right\}$. Accordingly, there is a constant $k>0$ so that any conjugacy between words $u_{0}, u_{1}$ in the generators of $\pi_{1} X$ can be realized by a sequence of moves $u_{t} \mapsto a_{t} u_{t} a_{t}^{-1}$ where $a_{t}$ is a generator and $d\left(1, u_{t}\right) \leq K \max \left\{\left|u_{0}\right|,\left|u_{1}\right|\right\}$; see [35] p.445. This control carries 
over to $\mathrm{SH}$ and bicombable groups, where it yields a solution to the conjugacy problem. But control is lost as one weakens the link to $\mathrm{CAT}(0)$ spaces [27].

Theorem 3.3. The conjugacy problem is unsolvable in certain combable groups.

Zlil Sela [88] solved the isomorphism problem for torsion-free hyperbolic groups. His solution was recently extended to a large class of relatively hyperbolic groups by F. Dahmani and D. Groves [48]. When combined with the topological rigidity theorem of T. Farrell and L. Jones [56], Sela's result implies that the homeomorphism problem is solvable among closed $n$-manifolds, $n \geq 5$, that admit a metric of negative curvature. The results of Farrell and Jones remain valid for non-positively curved manifolds and there is therefore considerable interest in the isomorphism problem in $\mathrm{SH}, \mathcal{C}_{0}$ and the subclass consisting of the fundamental groups of such manifolds. These problems remain open, but beyond $\mathrm{SH}$ decidability is lost:

Theorem 3.4. [27] The isomorphism problem is unsolvable among combable groups.

To prove this one seeks a recursive sequence $\left\langle A \mid R_{n}\right\rangle$ of presentations of combable groups such that there is no algorithm that decides which are isomorphic. Starting with a hyperbolic group $H$ in which there is no algorithm to decide when maps $\phi_{n}: F_{r} \rightarrow H$ are epic, one extends $\phi_{n}$ to a homomorphism $\hat{\phi}_{n}: F_{2 r} \rightarrow H$ and defines $\left\langle A \mid R_{n}\right\rangle$ to be a certain presentation of $\Gamma *_{\Sigma(n)} \Gamma$, where $\Gamma=\left(\mathbb{Z}_{2} * H\right) \times F_{2 r}$ and $\Sigma(n)=\left\{\left(\hat{\phi}_{n}(x), x\right) \mid x \in F_{2 r}\right\}$ is quasi-isometrically embedded.

3.5. Asynchronous combings and 3-manifolds. As one moves further from the $\operatorname{CAT}(0)$ setting and weakens the convexity condition on the metric, combings arise that only satisfy a weakened form of the fellow-traveller property (3.1): the paths $\sigma_{\gamma}$ remain close only after monotone reparameterization.

Little of the strength of non-positive curvature remains in this definition but it does embrace a much larger class of groups, e.g. [22, 55]. Moreover, the amount of convexity retained is enough to provide a reasonable solution to the word problem and to ensure that these groups have classifying spaces with only finitely many cells in each dimension. Epstein et al. examined what happens when one requires $\sigma(\Gamma)$ to be regular. Bob Gilman [32] and I explored larger families of languages.

Epstein and Thurston [55] proved that the fundamental group of a compact 3-manifold $M$ is automatic if and only if it satisfies a quadratic isoperimetric inequality (which excludes connected summands that are torus bundles over the circle with infinite holonomy). Gilman and I, building on [22], sharpened the negative part of their theorem and proved that by using indexed languages one can construct combings that encode the coarse geometry of any cocompact 3-manifold. (This result relies on the fact that 3-manifolds are geometrizable.)

Theorem 3.5. The fundamental group of every compact 3-manifold $M$ is asychronously Ind-combable, but in some cases $\pi_{1} M$ is not asychronously CF-combable. 


\section{Word problems and filling invariants}

4.1. Dehn functions. The word problem for finitely presented groups has been at the heart of combinatorial group theory since its inception. When one attacks the word problem for a finitely presented group $\Gamma=\langle\mathcal{A} \mid \mathcal{R}\rangle$ directly, one's chances of success depend heavily on the Dehn function $\delta_{\Gamma}: \mathbb{N} \rightarrow \mathbb{N}$. Given a word $w$ in the kernel of the map from the free group $F(\mathcal{A})$ to $\Gamma$, one defines

$$
\operatorname{Area}(w):=\min \left\{N \mid w \stackrel{\text { free }}{=} \prod_{i=1}^{N} x_{i}^{-1} r_{i} x_{i} \text { some } x_{i} \in F(\mathcal{A}), r_{i} \in \mathcal{R}^{ \pm 1}\right\}
$$

and

$$
\delta_{\Gamma}(n):=\max \left\{\operatorname{Area}(w)\left|w=_{\Gamma} 1,\right| w \mid \leq n\right\} .
$$

The subscript on $\delta_{\Gamma}$ is somewhat misleading since different finite presentations of the same group will in general yield different Dehn functions. This ambiguity is tolerated because it is tightly controlled: if the groups defined by two finite presentations are isomorphic, or just quasi-isometric, the corresponding Dehn functions are $\simeq$ equivalent in the following sense: given two monotone functions $f, g:[0, \infty) \rightarrow[0, \infty)$, one writes $f \preccurlyeq g$ if there exists a constant $C>0$ such that $f(l) \leq C g(C l+C)+C l+C$ for all $l \geq 0$, and $f \simeq g$ if $f \preccurlyeq g$ and $g \preccurlyeq f$; and one extends this relations to include functions $\mathbb{N} \rightarrow[0, \infty)$.

If $\delta_{\Gamma}(n) \preccurlyeq n^{d}$, one says that $\Gamma$ satisfies a polynomial isoperimetric inequality of degree $d$. See [30] for references and basic facts about Dehn functions.

The first step from word problems to filling problems is provided by van Kampen's Lemma [70], which states that $\operatorname{Area}(w)$ is equal to the least number of 2-cells in any van Kampen diagram for $w$. Such a diagram describes a combinatorial filling (i.e. null-homotopy) for the loop labelled $w$ in the 1-skeleton of the 2-complex $K=K(\mathcal{A}, \mathcal{R})$ described in the first paragraph of the introduction.

Suppose $\Gamma=\langle\mathcal{A} \mid \mathcal{R}\rangle$ acts properly and cocompactly by isometries on a Riemannian manifold $X$. Fix $p \in X$. If $X$ is simply connected, the quasi-isometry $\gamma \mapsto \gamma \cdot p$ extends to a $\Gamma$-equivariant map $\phi: \widetilde{K} \rightarrow X$. An edge-loop $\sigma$ in $\mathcal{C}_{\mathcal{A}}(\Gamma)=\widetilde{K}^{(1)}$ defines a piecewise-geodesic loop $\phi \circ \sigma$ in $X$, and a van Kampen diagram that describes a filling of $\sigma$ defines a singular disc in $X$ with boundary $\phi \circ \sigma$. Conversely, any rectifiable loop $c$ in $X$ can be approximated by a word-like loop $\phi \circ \sigma$ whose length is linearly bounded by that of $c$ and (more delicately) disc-fillings $g: \mathbb{D}^{2} \rightarrow X$ with $\left.g\right|_{\partial D}=c$ give rise to van Kampen diagrams for $\sigma$.

This line of thought, initiated by Gromov, suggests that the large-scale behaviour of Riemannian filling-discs - quantified by features such as area, radius, diameter etc. - should be translated to the setting of van Kampen diagrams. Then, in the spirit of van Kampen's Lemma, these features can be used to measure the complexity of the word problem in $\Gamma$. The following implementation of this strategy was described by Gromov and presented in detail in [30] (also [45]).

Let $M$ be a compact Riemannian manifold with universal cover $\widetilde{M}$. Define the filling area FArea $(c)$ of a rectifiable loop $c: S^{1} \rightarrow \widetilde{M}$ to be the infimum of the 
areas of all Lipschitz maps $g: \mathbb{D} \rightarrow X$ where $\mathbb{D}$ is the standard 2-disc and $\left.g\right|_{\partial D}$ is a monotone reparameterization of $c$. Consider

$$
\operatorname{Fill}_{M}(l):=\sup \left\{\operatorname{FArea}(c) \mid c: S^{1} \rightarrow \widetilde{M} \text { length }(c) \leq l\right\},
$$

the genus zero, 2-dimensional, isoperimetric function of $M$.

Theorem 4.1 (Filling Theorem). Fill $_{M}(l) \simeq \delta_{\pi_{1} M}(l)$.

A similar statement holds for isoperimetric functions of more general compact spaces with upper curvature bounds. Similar theorems also hold with area replaced by other invariants of the geometry of filling-discs. Among these, the most actively studied is intrinsic diameter (i.e. diameter measured in the induced length metric on the disc); in this case, FArea and the Dehn function are replaced by (intrinsic) isodiametric functions. When translated into algebra, bounds on intrinsic diameter correspond to bounds on the length of the conjugating words $x_{i}$ in (4.1).

Results giving lower bounds on intrinsic diameter often proceed via extrinsic diameter, i.e. diameter measured in the metric on the ambient space. It was only recently that Tim Riley and I constructed the first compact manifolds for which the isodiametric functions corresponding to the choice intrinsic-versus-extrinsic have distinct asymptotic behaviour [43]. This extends a considerable body of work relating different aspects of the geometry of filling discs [64, 84].

4.2. The Isoperimetric Spectrum. A major theme in geometric group theory in the 1990s and into this century has been the struggle to determine which $\simeq$ classes of functions arise as Dehn functions. (I shall say little about the complementary challenge of calculating the Dehn functions of groups of special interest.)

The development of our knowledge can be charted by how the set

$$
\mathrm{IP}=\left\{\rho \in[1, \infty) \mid f(n)=n^{\rho} \text { is } \simeq \text { a Dehn function }\right\}
$$

came to be understood. This set is called the isoperimetric spectrum. I should emphasize that it is far from the case that all Dehn functions are of the form $n^{\alpha}$ : there are non-polynomial Dehn functions such as $n^{\alpha} \log n$, as well as examples of small presentations with huge Dehn functions, e.g. faster than any iterated exponential (see 4.4). If $\Gamma$ has unsolvable word problem, $\delta_{\Gamma}(n)$ will grow faster than any recursive function (indeed this serves as a definition of such groups).

The class of groups with linear Dehn functions coincides with the class of hyperbolic groups. The non-hyperbolic groups in $\mathcal{C}_{0}$ and Auto have quadratic Dehn functions. Certain combable groups have cubic Dehn functions (3.2), as does the 3 -dimensional Heisenberg group. In about 1992, sequences of groups $\left(\Gamma_{d}\right)_{d \in \mathbb{N}}$ such that the Dehn function of $\Gamma_{d}$ is polynomial of degree $d$ were discovered by Gromov [64], Baumslag-Miller-Short [7], and Bridson-Pittet [42]. The literature now contains such sequences with all manner of additional properties. An example of a group whose Dehn function is polynomial of degree $d+1$ is $\mathbb{Z}^{d} \rtimes_{\phi} \mathbb{Z}$, where $\phi \in \mathrm{GL}(d, \mathbb{Z})$ has 1 s on the diagonal and superdiagonal and zeroes elsewhere.

A result of Gromov [65], reproved by many people, states that if the Dehn function of a group is sub-quadratic (i.e. $\delta_{\Gamma}(n)=o\left(n^{2}\right)$ ) then $\delta_{\Gamma}(n) \simeq n$. Thus 
IP $\cap(1,2)$ is empty. This begs the question of what other gaps there may be in IP, and whether there are any non-integral exponents at all. I settled this last question by constructing the abc groups of [20], formed by taking three torus bundles over the circle (different dimensions) and amalgamating their fundamental groups along central cyclic subgroups. Indiscrete families of exponents were first constructed in [86]. Noel Brady and I [15] completed the understanding of the coarse structure of IP by constructing a dense set of exponents in $[2, \infty)$.

Theorem 4.2. The closure of IP is $\{1\} \cup[2, \infty)$.

We proved this by associating to each pair of positive integers $p \geq q$ an aspherical 2-complex whose fundamental group

$$
G_{p, q}=\left\langle a, b, s, t \mid[a, b]=1, s a^{q} s^{-1}=a^{p} b, t a^{q} t^{-1}=a^{p} b^{-1}\right\rangle,
$$

has Dehn function $\simeq n^{2 \log _{2}(2 p / q)}$. These complexes are obtained by attaching a pair of annuli to a torus in a manner that ensures the existence of a family of discs in the universal cover that display a certain snowflake geometry. With Max Forester and Ravi Shankar [16], we developed a more sophisticated version of the snowflake construction that yields a much larger class of isoperimetric exponents, showing in particular that $[2, \infty) \cap \mathbb{Q} \subseteq$ IP.

Once one knows that IP is not a discrete set, one assumes that it will follow the general pattern of group theory by exhibiting all plausible levels of complexity. This expectation is realized in a remarkable piece of work by M. Sapir, J-C. Birget and E. Rips [86] who give a comprehensive description of Dehn functions $\delta_{\Gamma}(n) \succcurlyeq n^{4}$ by encoding the time functions of Turing machines. (The fine structure of $\operatorname{IP} \cap(2,4)$ has yet to be determined.) In a subsequent work with A.Yu. Ol'shanskii [19] the same authors prove that the word problem for $\Gamma$ is in NP if and only if $\Gamma$ is a subgroup of a finitely presented group with polynomial Dehn function.

4.3. Groups with quadratic Dehn functions. The structure of IP provides us with two classes of groups that demand special attention - the groups with linear Dehn functions (which we know to be the hyperbolic groups) and the groups with quadratic Dehn functions. It is far from clear what to expect from groups in this second class. They have simply-connected asymptotic cones [80] but so do many (not all [23]) other groups with polynomial Dehn functions. It is unknown if they all have a solvable conjugacy problem. IP(2) contains many nilpotent groups $N$ that are not virtually abelian and certain non-nilpotent polycyclic groups [53]. It is unknown if it contains any solvable groups that are not virtually polycyclic. Thurston proposed that $\mathrm{SL}(n, \mathbb{Z}), n \geq 4$, should be in $\operatorname{IP}(2)$ but this has not been confirmed. V. Guba [67] proved that Richard Thompson's group (which is torsion-free, of type $\mathrm{FP}_{\infty}$, and infinite dimensional) lies in $\operatorname{IP}(2)$. Groves and I proved the same for groups of the form $F_{n} \rtimes \mathbb{Z}$ [33].

4.4. Applications to the geometry and topology of manifolds. The dictionary of equivalence illustrated by the Filling Theorem translates information about Dehn functions into statements about the geometry of manifolds. 
But there are also less obvious mechanisms that allow one to gain geometric and topological information from an understanding of the nature of Dehn functions.

The Andrews-Curtis conjecture is one of the famous open problems of lowdimensional topology. It is related to the Zeeman conjecture and the smooth 4-dimensional Poincaré conjecture [72]. It asserts that one can reduce any balanced presentation $\left\langle a_{1}, \ldots, a_{n} \mid r_{1}, \ldots, r_{n}\right\rangle$ of the trivial group to the presentation $\left\langle a_{1}, \ldots, a_{n} \mid a_{1}, \ldots, a_{n}\right\rangle$ by a sequence of certain elementary moves. The main construction of [31] associates a balanced presentation $P_{w}$ to each word $w$ in the generators of a group $B$ satisfying a deletion condition. $P_{w}$ presents the trivial group if and only if $w=1$ in $B$. Moreover, if $P_{w}$ presents $\{1\}$ then it satisfies the Andrews-Curtis conjecture but the number of elementary moves required to trivialise it is bounded below by $\log \operatorname{Area}_{B}^{*}(w)$.

One gets dramatic lower bounds by taking $B=\left\langle a, t \mid\left[t^{-1}, a\right]=a^{r-1}\right\rangle$, since $\delta_{B}(n) \simeq \Delta_{r}\left\lfloor\log _{2} n\right\rfloor$ where $\Delta_{r}(m)$ is defined by $\Delta_{r}(1):=r$ and $\Delta_{r}(m+1):=$ $r^{\Delta_{r}(m)}$. In this case $P_{w}$ has 4 generators and relations of total length $2(10+|w|+r)$.

In a remarkable series of papers, A. Nabutovsky and S. Weinberger [78] use Dehn functions to explore the sub-level sets of functionals such as diameter on moduli spaces of metrics for closed manifolds $M^{n}, n \geq 5$. The constructions in [31] allow one to extend parts of their work to dimension 4 .

4.5. Higher-dimensional isoperimetric inequalities. In the Riemannian context, having considered the isoperimetric problem for discs filling loops, it is natural to explore fillings of higher-dimensional spheres. In particular one wants to understand the isoperimetric function that bounds the volume of optimal ball-fillings. Correspondingly, one defines the $k$-th order Dehn function $\delta^{(k)}$ of a finitely presented group $\Gamma$ that has a classifying space $X$ with a compact $(k+1)$-skeleton $X^{(k+1)}$. Such functions were introduced by Gromov [64]. Roughly speaking $\delta^{(k)}(l)$ bounds the number of $(k+1)$-cells required to fill any singular $k$-sphere in $X^{(k)}$ comprised of at most $l k$-cells. The algebraic foundations of the subject were worked out carefully by Alonso et al. [3] and interpreted more topologically in [24]. From an algebraic point of view, $\delta^{(k)}(l)$ provides the least upper bound on the number of summands required to express an element $[f] \in \pi_{k}\left(X^{(k)}\right)$ as a $\Gamma$-linear combination of the attaching maps of the $(k+1)$-cells of $X$. The $\simeq$ equivalence class of $\delta^{(k)}$ is an invariant of quasi-isometry.

In each dimension $k$ one has the isoperimetric spectrum

$$
\operatorname{IP}^{(k)}=\left\{\alpha \in[1, \infty) \mid f(x)=x^{\alpha} \text { is } \simeq \text { a } k \text {-th order Dehn function }\right\} .
$$

Until recently, our knowledge even for $\mathrm{IP}^{(2)}$ was remarkably sparse, but my recent work with Brady, Forester and Shankar [16] remedies this. We prove that if $P$ is an irreducible non-negative integer matrix with Perron-Frobenius eigenvalue $\lambda>1$, and $r$ is an integer greater than every row sum in $P$, then for every $k \geq 2$ there is a group $\Gamma=\Sigma^{k-1} G_{r, P}$ with a compact $(k+1)$-dimensional classifying space such that $\delta^{(k)}(x) \simeq x^{2 \log _{\lambda}(r)}$. It follows from this and a related result in [16] that $\operatorname{IP}^{(k)}$ is dense in the range $[1+1 / k, \infty)$. Indeed the case of $1 \times 1$ matrices alone leads to:

Theorem 4.3. $\mathbb{Q} \cap\left[1+\frac{1}{k}, \infty\right) \subset \mathrm{IP}^{(k)}$. 
The exponent $1+1 / k$ arises for $\mathbb{Z}^{k+1}$. Comparing with $\mathrm{IP}=\mathrm{IP}^{(1)}$, it is tempting to speculate that $\overline{\mathrm{IP}}^{(k)}=\{1\} \cup[1+1 / k, \infty)$ but there are reasons to doubt this. One suspects that the fine structure of $\mathrm{IP}^{(k)}$ is similar to that of $\mathrm{IP}^{(1)}$.

The group $G_{r, P}$ is the fundamental group of an aspherical 2-complex $X_{r, P}$ assembled from a finite collection of annuli and tori; the rational number $r$ encodes the multiplicities of the attaching maps while the matrix $P$ encodes a prescription for the number and orientation of the tubes connecting each pair of tori. Leastarea discs in $\widetilde{X}_{r, P}$ exhibit a more subtle form of the snowflake geometry from [15]. When $r$ is an integer, certain families of these discs admit a precise scaling by a factor of $r$. One stacks scaled copies of them to form embedded 3-balls in the universal covering of the mapping torus associated to a certain 2-letter HNN extension $\Sigma G_{r, P}$ of $G_{r, P}$. These balls provide a lower bound on $\delta^{(2)}$ of $\Sigma G_{r, P}$; this proves to be sharp. The balls inherit the scaling property, so one can iterate.

The calculation of $\delta^{(k)}$ for $\Sigma^{(k-1)} G_{r, P}$ involves an induction on dimension. In order to make this work smoothly, one must bound not only the isoperimetric behaviour of disc-fillings for spheres but also the isoperimetric behaviour of other pairs of compact manifolds $(M, \partial M)$ mapping to the complexes in question. The topological approach to $\delta^{(k)}$ taken in [24] is well-adapted to such generalizations.

The homological filling invariants of the groups $\Sigma^{(k-1)} G_{r, P}$ exhibit a similar range of behaviour. Such invariants provide upper bounds on the size of (cellular) $(k+1)$-chains needed to fill $k$-cycles in the universal covering of a classifying space with finite $(k+1)$-skeleton; size is measured using the $\ell_{1}$-norm associated to the cellular basis. These invariants are easier to work with than their homotopical counterparts and relate well to the Riemannian setting - see [64] and [55] chap. 10.

\section{Subdirect products of hyperbolic groups}

The results in this section highlight a dichotomy in the behaviour of the finitely presented subgroups of direct products of hyperbolic groups: in general the structure of such subgroups can be fiendishly complicated; but for free groups and limit groups, these subgroups are remarkably controlled.

5.1. Encoding wildness. E. Rips [85] found a simple algorithm that associates to a finite presentation $\mathcal{Q}$ a short exact sequence $1 \rightarrow N \rightarrow H \rightarrow Q \rightarrow 1$, where $Q$ is the group that $\mathcal{Q}$ presents, $N$ is a 2-generator group, and $H$ is a 2 dimensional hyperbolic group. To get $H$ from $\mathcal{Q}$, one adds two new generators $a_{1}, a_{2}$, replaces the relations $r=1$ of $\mathcal{Q}$ by relations $r=U_{r}\left(a_{1}, a_{2}\right)$ and adds a new relation $x_{i}^{-\varepsilon} a_{j} x_{i}^{\varepsilon}=V_{i, j, \varepsilon}\left(a_{1}, a_{2}\right)$ for each generator $x_{i}$ in $\mathcal{Q}$ and $j=1,2, \varepsilon= \pm 1$; the words $U_{r}$ and $V_{i, j, \varepsilon}$ are chosen to satisfy a small-cancellation condition. This construction depends on the specific presentation $\mathcal{Q}$, not just the group $Q$.

The flexibility of the Rips construction is such that (at the expense of increasing the number of generators of $N$ ) one can arrange for $H$ to have additional properties such as being the fundamental group of a compact negatively curved 2-complex 
[93], [35] p.225, or residually finite [94]. Thus one can encode all of the complexity of finite group-presentations (the lions of figure 1) into the finitely generated subgroups of such $H$. But such constructions say nothing about finitely presented subgroups because, by a theorem of R. Bieri [14], $N$ is not finitely presentable if $Q$ is infinite. The following theorem from [6] obviates this difficulty.

Theorem 5.1 (1-2-3 Theorem). Suppose that $1 \rightarrow N \rightarrow \Gamma \rightarrow Q \stackrel{p}{\rightarrow} 1$ is exact. If $N$ is finitely generated, $\Gamma$ is finitely presented and $Q$ is of type $\mathrm{F}_{3}$, then the fibre-product $P:=\left\{\left(\gamma_{1}, \gamma_{2}\right) \mid p\left(\gamma_{1}\right)=p\left(\gamma_{2}\right)\right\} \subseteq \Gamma \times \Gamma$ is finitely presented.

The name of this theorem comes from the fact that the groups $N, \Gamma, Q$ are assumed to be of type $\mathrm{F}_{1}, \mathrm{~F}_{2}$ and $\mathrm{F}_{3}$ respectively. (Recall that a group $G$ is of type $\mathrm{F}_{k}$ if there exists a $K(G, 1)$ with compact $k$-skeleton.) The $\mathrm{F}_{3}$ hypothesis says $\pi_{2}$ of a presentation 2-complex for $Q$ is finitely generated as a $\mathbb{Z} Q$-module. This allows one to control the relations among the generators of $N \times N$ (cf. [4]).

By combining the Rips construction and the 1-2-3 Theorem, one can encode the complexities of arbitrary finitely presented groups directly into the structure of finitely presented subgroups of direct products of hyperbolic groups.

An application of this principle is described it the next section. Several other applications are given in [6], one of which was refined in [40] to prove that there exist 2-dimensional hyperbolic groups $\Gamma$ such that there is no algorithm to decide isomorphism among the finitely presented subgroups of $\Gamma \times \Gamma \times \Gamma$.

5.2. Subdirect products of surface Groups. John Stallings [89] and Robert Bieri [13] showed that among the kernels of maps from direct products of free groups to abelian groups one finds a range of finiteness properties; in particular there exist finitely presented subgroups of $F_{2} \times F_{2} \times F_{2}$ whose third homology is not finitely generated and finitely presented subgroups $S$ of a direct product of $n$ free groups that are of type $\mathrm{F}_{n-1}$ with $H_{n}(S, \mathbb{Z})$ not finitely generated. Thus one senses that the wild behaviour observed above may continue among subdirect products of free groups, and indeed it does for finitely generated subgroups [76].

But Gilbert Baumslag and Jim Roseblade proved that the only finitely presented subgroups $S$ of a direct product of two free groups are the obvious ones: such $S$ are free or have a subgroup of finite index that is the product of its intersections with the factors. Howie, Miller, Short and I [39] discovered an analogous phenomenon in higher dimensions, cf. (5.3).

If a subgroup $S$ of a direct product of $n$ free and surface groups is of type ${ }^{4} \mathrm{~F}_{n}$ then $S$ has a subgroup of finite index that is a direct product of free and surface groups.

The case of surface groups is important because of its implications concerning the fundamental groups of compact Kähler manifolds. The work of Delzant and Gromov [52] shows that if such a group $\Gamma$ is torsion-free and has sufficient multiended splittings, then there is an exact sequence $1 \rightarrow \mathbb{Z}^{n} \rightarrow \Gamma_{0} \rightarrow S \rightarrow 1$, where $S$ is a subdirect product of surface groups and $\Gamma_{0} \subset \Gamma$ has finite index. Motivated by this, one would like to understand all finitely presented subdirect products of

\footnotetext{
${ }^{4}$ It is enough that finite-index subgroups of $S$ have $H_{i}(-, \mathbb{Z})$ finitely generated for $i \leq n$.
} 
surface groups. In [41] Miller and I proved the following theorem and a weaker version (involving nilpotent quotients) for products of arbitrarily many surfaces.

Theorem 5.2. If $S$ is a finitely presented subgroup of a direct product of at most three surface groups, then either $S$ is virtually a product of free and surface groups (the case where $S$ is of type $\mathrm{F}_{3}$ ) or else $G$ is virtually the kernel of a map from a product of surface groups to an abelian group (the Stallings-Bieri situation).

One hopes that a complete classification of the finitely presented subdirect products of free and surface groups may be within reach. What we have already proved shows that the conjugacy and membership problems are solvable for all finitely presented subgroups of direct products of surface groups. This would not remain true if one were to replace surface groups by arbitrary 2-dimensional hyperbolic groups or Kleinian groups. Likewise, the splitting phenomenon for subgroups of type $\mathrm{F}_{n}$ does not extend to these classes. But it is does extend to limit groups.

5.3. Limit groups again. A finitely generated group $L$ is fully residually free if for each finite subset $X \subset L$ there is a homomorphism to a finitely generated free group $\psi_{X}: L \rightarrow F$ that is injective on $X$. It is difficult to prove that such $L$ are finitely presented but it is then easy to deduce that these are the limit groups defined in (1.2). The term limit group was coined by Sela to connote that these are the groups that occur as limits of stable sequences $\phi_{n}: G \rightarrow F$, where $G$ is an arbitrary finitely generated group and stable means that for each $g \in G$ either $I_{g}=\left\{n \in \mathbb{N}: \phi_{n}(g)=1\right\}$ or $J_{g}=\left\{n \in \mathbb{N}: \phi_{n}(g) \neq 1\right\}$ is finite; the limit is the quotient of $G$ by $\left\{g|| I_{g} \mid=\infty\right\}$.

Such sequences arise when one studies $\operatorname{Hom}(G, F)$. A homomorphism $\phi: G \rightarrow$ $F$ gives an action of $G$ on the tree that it the Cayley graph of $F$, and it is profitable to examine sequences $\left(\phi_{n}\right)$ in the space of $G$-actions on $\mathbb{R}$-trees. By bringing to bear much of what is known about such spaces, Sela ([87] et seq.) obtains a finite parameterization of $\operatorname{Hom}(G, F)$ and a hierarchical decomposition of limit groups. His description of the elementarily free groups $\mathrm{EF} \subset \mathcal{L}$ solves a famous problem of A. Tarski. Similar results were obtained in a parallel project by O. Kharlampovich and A. Myasnikov [71]. For an introduction to limit groups, see [10].

Jim Howie and I [37, 38] and H. Wilton [92] have been using Sela's work to explore the subgroup structure of limit groups and their direct products. The similarities with surface groups include:

Theorem 5.3. [37] If $G_{1}, \ldots, G_{n}$ are elementarily free and $\Gamma \subset G_{1} \times \cdots \times G_{n}$ is of type $\mathrm{FP}_{\mathrm{n}}$, then there are finitely presented subgroups $H_{i} \subset G_{i}$ such that $\Gamma$ is isomorphic to a finite-index subgroup of $H_{1} \times \cdots \times H_{n}$.

It is likely that this can be extended from EF to $\mathcal{L}$ as conjectured by Sela.

\section{Two questions of Grothendieck}

In this section I shall outline my solution with Fritz Grunewald to two problems concerning profinite completions and representations of groups that were posed by 
Alexander Grothendieck in 1970 [66]. The proof exemplifies two general points that I made earlier: the importance of being able to craft groups with specific properties, and the usefulness of the encodings into subdirect products.

6.1. Profinite completions. The profinite completion of a group $\Gamma$ is the inverse limit of the directed system of finite quotients of $\Gamma$; it is denoted by $\hat{\Gamma}$. If $\Gamma$ is residually finite, the natural map $\Gamma \rightarrow \hat{\Gamma}$ is injective. In [66] Grothendieck related the representation theory of a finitely generated group to its profinite completion: if $A \neq 0$ is a commutative ring and $u: \Gamma_{1} \rightarrow \Gamma_{2}$ is a homomorphism of finitely generated groups, then $\hat{u}: \hat{\Gamma}_{1} \rightarrow \hat{\Gamma}_{2}$ is an isomorphism if and only the restriction functor $u_{A}^{*}: \operatorname{Rep}_{A}\left(\Gamma_{2}\right) \rightarrow \operatorname{Rep}_{A}\left(\Gamma_{1}\right)$ is an equivalence of categories, where $\operatorname{Rep}_{A}(\Gamma)$ is the category of finitely presented $A$-modules with a $\Gamma$-action.

Grothendieck investigated under what circumstances $\hat{u}: \hat{\Gamma}_{1} \rightarrow \hat{\Gamma}_{2}$ being an isomorphism implies that $u$ is an isomorphism. This led him to pose the following problem: Let $\Gamma_{1}$ and $\Gamma_{2}$ be finitely presented, residually finite groups and let $u$ : $\Gamma_{1} \rightarrow \Gamma_{2}$ be a homomorphism such that $\hat{u}: \hat{\Gamma}_{1} \rightarrow \hat{\Gamma}_{2}$ is an isomorphism of profinite groups. Does it follow that $u$ is an isomorphism from $\Gamma_{1}$ onto $\Gamma_{2}$ ?

A negative solution to the corresponding problem for finitely generated groups was given by Platonov and Tavgen [81]. But there is an emphasis on finite presentability in Grothendieck's problem because of his original motivation for studying profinite completions: he wanted to understand the extent to which the topological fundamental group of a complex projective variety determines the algebraic fundamental group, and vice versa. In the Spring of 2003 Fritz Grunewald and I settled Grothendieck's question in the negative.

Theorem 6.1. There exist residually finite, 2-dimensional, hyperbolic groups $H$ and finitely presented subgroups $P \subseteq \Gamma:=H \times H$ of infinite index, such that $P$ is not abstractly isomorphic to $\Gamma$, but $u: P \hookrightarrow \Gamma$ induces an isomorphism $\hat{u}: \hat{P} \rightarrow \hat{\Gamma}$.

The first ingredient in the proof is the following distillation of arguments of Platonov and Tavgen [81]. Let $1 \rightarrow N \rightarrow H \rightarrow Q \rightarrow 1$ be a short exact sequence of groups with fibre product $u: P \hookrightarrow H \times H$. If $Q$ is superperfect ${ }^{5}$ and has no finite quotients, and $H$ is finitely generated, then $\hat{u}: \hat{P} \rightarrow \hat{H} \times \hat{H}$ is an isomorphism.

One applies this criterion to the output of an algorithm obtained by combining D. Wise's refinement [94] of the Rips construction with the 1-2-3 Theorem [6]:

There is an algorithm that associates to any finite aspherical presentation $\mathcal{Q}$ a short exact sequence $1 \rightarrow N \rightarrow H \rightarrow Q \rightarrow 1$ and a finite presentation for the fibre product $P \subset H \times H$, where $H$ is hyperbolic and residually finite.

To complete the proof, one needs suitable input presentations $\mathcal{Q}$. A simple calculation in homology shows that if a perfect group has a balanced presentation then it is superperfect. Thus it suffices to construct balanced, aspherical presentations of infinite groups with no non-trivial finite quotients. The following example was constructed in [34]; earlier examples are due to G. Higman [69]. Let $p \geq 2$.

$\left\langle a_{1}, a_{2}, \hat{a}_{1}, \hat{a}_{2} \mid a_{1}^{-1} a_{2}^{p} a_{1} a_{2}^{-p-1}, \hat{a}_{1}^{-1} \hat{a}_{2}^{p} \hat{a}_{1} \hat{a}_{2}^{-p-1}, a_{1}^{-1}\left[\hat{a}_{2}, \hat{a}_{1}^{-1} \hat{a}_{2} \hat{a}_{1}\right], \hat{a}_{1}^{-1}\left[a_{2}, a_{1}^{-1} a_{2} a_{1}\right]\right\rangle$.

\footnotetext{
${ }^{5} H_{1}(Q, \mathbb{Z})=H_{2}(Q, \mathbb{Z})=0$
} 
At the expense of complicating the construction of the Grothendieck pair $P \hookrightarrow$ $\Gamma \times \Gamma$, one can replace the requirement that the input presentation $\mathcal{Q}$ be aspherical by the hypothesis that $Q$ be of type $\mathrm{F}_{3}$. This allows one to associate a Grothendieck pair to any group of type $\mathrm{F}_{3}$, for if $\mathcal{G}$ is a class (such as $\mathrm{F}_{3}$ ) closed under the formation of HNN extensions and amalgamated free products along free groups, one can embed any group $G \in \mathcal{G}$ into a $\bar{G} \in \mathcal{G}$ that has no finite quotients [28].

6.2. Grothendieck's Tannaka duality groups. In the same paper [66] as he raised the problem described above, Grothendieck described an idea for reconstructing a residually finite group from the tensor product structure of its representation category $\operatorname{Rep}_{A}(\Gamma)$. He encoded this structure into a Tannaka duality group: if $\operatorname{Mod}(A)$ is the category of all finitely generated $A$-modules and $\mathcal{F}: \operatorname{Rep}_{A}(\Gamma) \rightarrow \operatorname{Mod}(A)$ is the forgetful functor, Grothendieck defines $\mathrm{cl}_{A}(\Gamma)$ to be the group of natural self-transformations of the functor $\mathcal{F}$ that are compatible with the tensor product $\otimes_{A}$. And he poses the following problem: If $\Gamma$ is a finitely presented, residually finite group, is the natural monomorphism from $\Gamma$ to $\mathrm{cl}_{A}(\Gamma)$ an isomorphism for every non-zero commutative ring $A$, or at least some suitable commutative ring $A \neq 0$ ?

Theorem 6.2. [34] If $P$ is one of the groups constructed in Theorem 6.1, then $P$ is of infinite index in $\mathrm{cl}_{A}(P)$ for every commutative ring $A \neq 0$.

Previously, Alex Lubotzky [75] had exhibited finitely presented, residually finite groups $\Gamma$ such that $\Gamma \rightarrow \operatorname{cl}_{A}(\Gamma)$ is not surjective when $A=\mathbb{Z}$.

\section{References}

[1] Alibegović, E., Bestvina, M., Limit groups are CAT(0). J. London Math. Soc., in press. ArXiv math.GR/0410198.

[2] Alonso, J., Bridson, M.R., Semihyperbolic groups. Proc. London Math. Soc. 70 (1995), 56-114.

[3] Alonso, J.M.; Wang, X.; Pride, S.J., Higher-dimensional isoperimetric (or Dehn) functions of groups. J. Group Theory 2 (1999), 81-112.

[4] Baik, Y. G.; Harlander, J.; Pride, S. J., The geometry of group extensions. J. Group Theory 1 (1998), 395-416.

[5] Ballmann W., Lectures on spaces of nonpositive curvature. DMV Seminar 25, Birkhäuser Verlag, Basel, 1995.

[6] Baumslag G., Bridson M.R., Miller C.F., Short H., Fibre products, non-positive curvature and decision problems. Comm. Math. Helv. 75 (2000), 457-477.

[7] Baumslag, G., Miller, C.F., Short, H., Isoperimetric inequalities and the homology of groups. Invent. Math. 113 (1993), 531-560.

[8] Baumslag, G., Roseblade, J.E., Subgroups of the direct product of two free groups. J. London Math. Soc., 30 (1984), 44-52.

[9] Bestvina M., The topology of Out $\left(F_{n}\right)$. Proc. ICM, Bejing 2002, vol. II, 373-384 Higher Education Press (China). 
[10] Bestvina M., Feighn M., Notes on Sela's work: Limit groups and MakaninRazborov diagrams. To appear in Geometric and cohomological methods in group theory (M.R. Bridson, P.H. Kropholler, I.J. Leary, eds.).

[11] Bestvina M., Feighn M., Handel M., The Tits alternative for Out $\left(F_{n}\right)$ II: A Kolchin type theorem. Ann. of Math., 161 (2005), 1-59.

[12] Bestvina M., Handel, M., Train tracks and automorphisms of free groups. Ann. of Math., 135 (1992), 1-51.

[13] Bieri R., Normal subgroups in duality groups and in groups of cohomological dimension 2. J. Pure Appl. Algebra 7 (1976), 35-51.

[14] Bieri R., Homological dimension of discrete groups. Queen Mary Math. Notes (1976).

[15] Brady,N., Bridson, M.R., There is only one gap in the isoperimetric spectrum. GAFA (Geom. Funct. Anal.) 10 (2000), 1053-1070.

[16] Brady N., Bridson, M.R., Forester M., Shankar K., Snowflake groups, PerronFrobenius exponents and isoperimetric spectra. Preprint 2006.

[17] Brady N., Bridson, M.R., Reeves, L., Free-by-cyclic groups that are not automatic. Preprint 2006.

[18] Brady, N., Crisp, J., Two-dimensional Artin groups with CAT(0) dimension three. Geom. Dedicata 94 (2002), 185-214.

[19] Birget, J-C., Olshanskii, A.Yu., Rips, E., Sapir, M.V., Isoperimetric functions of groups and computational complexity of the word problem. Ann. of Math. 156 (2002), 467-518.

[20] Bridson, M.R., Fractional isoperimetric inequalities and subgroup distortion. $J$. Amer. Math. Soc. 12 (1999), 1103-1118.

[21] Bridson, M.R., On the geometry of normal forms in discrete groups. Proc. London Math. Soc., 67 (1993), 516-616.

[22] Bridson, M.R., Combing semidirect products and 3-manifold groups. GAFA (Geom. and Funct. Anal.) 3 (1993), 263-278.

[23] Bridson M.R., Asymptotic cones and polynomial isoperimetric inequalities. Topology 38 (1999), 543-554.

[24] Bridson, M.R., Polynomial Dehn functions and the length of asynchronous automatic structures. Proc. London Math. Soc. 85 (2002), 441-466.

[25] Bridson, M.R., Length functions, non-positive curvature and the dimension of discrete groups. Math. Res. Lett. 8 (2001), 557-567.

[26] Bridson, M.R., Combings of groups and the grammar of reparameterisation. Comment. Math. Helv. 78 (2003), 752-771.

[27] Bridson, M.R., The conjugacy and isomorphism problems for combable groups. Math. Ann. 327 (2003), 305-314.

[28] Bridson, M.R., Controlled embeddings into groups that have no non-trivial finite quotients, Geom. Topol. Monogr. 1 (1998), 99-116.

[29] Bridson, M.R., On the subgroups of semihyperbolic groups. In Essays on geometry and related topics, Vol. 1, Monogr. Enseign. Math. 38, Geneva, 2001, 85-111.

[30] M.R. Bridson, The geometry of the word problem. In Invitations to geometry and topology (M.R. Bridson, S.M. Salamon, eds.), Oxford University Press, 2002. 
[31] Bridson, M.R., On the complexity of balanced presentations and the Andrews-Curtis conjecture. Preprint 2006.

[32] Bridson, M.R., Gilman, R., Formal language theory and the geometry of 3-manifolds. Comm. Math. Helv. 71 (1996), 525-555.

[33] Bridson, M.R., Groves, D.P., The quadratic isoperimetric inequality for mapping tori of free group automorphisms I and II. ArXiv math.GR/0211459 and GR/0507589.

[34] Bridson, M.R., Grunewald F., Grothendieck's problems concerning profinite completions and representations of groups, Ann. of Math. 160 (2004), 359-373.

[35] Bridson, M.R., Haefliger A., Metric spaces of non-positive curvature. Grundlehren der Math. Wiss., Vol. 319, Springer-Verlag, Heidelberg, 1999.

[36] Bridson, M.R., Howie J., Conjugacy of finite subsets in hyperbolic groups, Intl. J. Alg. Comp. 15 (2005), 725-756.

[37] Bridson, M.R., Howie J., Subgroups of direct products of elementarily free groups. ArXiv math.GR/0506482.

[38] Bridson, M.R., Howie J., Subgroups of direct products of two limit groups. ArXiv math.GR/0510353.

[39] Bridson, M.R., Howie J., Miller C.F., Short H., The subgroups of direct products of surface groups. Geom. Dedicata 92 (2002), 95-103.

[40] Bridson, M.R., Miller C.F., Recognition of subgroups of direct products of hyperbolic groups. Proc. Amer. Math. Soc. 132 (2004), 59-65.

[41] Bridson, M.R., Miller C.F., Structure and finiteness properties for subdirect products of groups, Preprint 2006.

[42] Bridson, M.R., Pittet C., Isoperimetric inequalities for the fundamental groups of torus bundles over the circle, Geom. Dedicata, 49 (1994), 203-219.

[43] Bridson, M.R., Riley T., Intrinsic versus extrinsic diameter for Riemannian filling discs and van Kampen diagrams. ArXiv math.GR/0511004.

[44] Bridson, M.R., Vogtmann K., Automorphism groups of free groups, surface groups and free abelian groups. ArXiv math.GR/0507612.

[45] Burillo, J., Taback, J., Equivalence of geometric and combinatorial Dehn functions. New York J. Math. 8 (2002), 169-179.

[46] Cannon, J.W., The combinatorial structure of cocompact discrete hyperbolic groups. Geom. Dedicata 16 (1984), 123-148.

[47] Champetier C., Guirardel V.; Limit groups as limits of free groups: compactifying the set of free groups, Israel J. Math. 146 (2005), $1-76$.

[48] Dahmani F., Groves D., The isomorphism problem for toral relatively hyperbolic groups. ArXiv math.GR/0512605.

[49] Davis, M.W., Nonpositive curvature and reflection groups. In Handbook of geometric topology, 373-422, North-Holland, Amsterdam, 2002.

[50] Dehn, M., Über unendliche diskontinuierliche Gruppen. Math. Ann. 71 (1912), 116-144.

[51] Delzant T., Sous-groupes distingués et quotients des groups hyperboliques. Duke Math. J. 83 (1996), 661-682. 
[52] Delzant T., Gromov M., Cuts in Kähler groups. In Infinite Groups:Geometric, Combinatorial and Dynamical Aspects, Progress in Math. 248, Birkhäuser, Basel 2005.

[53] Druţu, C., Filling in solvable groups and in lattices in semisimple groups. Topology 43 (2004), no. 5, 983-1033.

[54] Druţu, C., Sapir, M.V., Tree-graded spaces and asymptotic cones of groups (with appendix by D. Osin and M. Sapir). Topology 44 (2005), no. 5, 959-1058.

[55] Epstein, D.B.A., Cannon, J.W., Holt, D.F., Levy, S.V.F., Paterson, M.S., Thurston, W.P., Word processing in Groups. A.K. Peters, Boston MA, 1992.

[56] Farrell, F.T., Jones, L.E., Topological rigidity for compact non-positively curved manifolds. In Differential geometry: Riemannian geometry (Los Angeles, CA, 1990), Proc. Symp. Pure Math. 54, Amer. Math. Soc., Providence, RI, 1993, 229-274

[57] Gersten, S.M., The automorphism group of a free group is not a $\operatorname{CAT}(0)$ group. Proc. Amer. Math. Soc. 121 (1994), 999-1002.

[58] Gersten, S.M., Dehn functions and $l_{1}$-norms for finite presentations. In Algorithms and Classification in Combinatorial Group Theory (G. Baumslag, C.F. Miller, eds.), MSRI Publ. 23 (1992), Springer-Verlag, New York.

[59] Gersten, S. M.; Short, H.B. Rational subgroups of biautomatic groups. Ann. of Math. 134 (1991), 125-158.

[60] Grigorchuk R.I., Degrees of growth of finitely generated groups and the theory of invariant means. Izv. Akad. Nauk SSSR Ser. Mat. 48 (1984), 939-985.

[61] Gromoll, D., Wolf, J., Some relations between the metric structure and the algebraic structure of the fundamental group in manifolds of non-positive curvature. Bull. Amer. Math. Soc. 77 (1971), 545-552.

[62] Gromov, M., Infinite groups as geometric objects. Proc. ICM, Warsaw 1978, PWN, Warsaw, 1984, 385-392.

[63] Gromov, M., Groups of polynomial growth and expanding maps. IHÉS Publ. Math. 53 (1981), 53-73.

[64] Gromov, M., Asymptotic invariants of infinite groups. Geometric Group Theory, Vol. 2 (G.A. Niblo, M.A. Roller, eds.), London Math. Soc. Lecture Notes 182, 1993.

[65] Gromov M., Hyperbolic groups. In Essays in Group Theory (S.M. Gersten, ed.), Springer-Verlag, New York, 1987, pp. 75-263.

[66] Grothendieck, A., Représentations linéaires et compactification profinie des groupes discrets. Manuscripta Math. 2 (1970), 375-396.

[67] Guba, V. S., The Dehn function of Richard Thompson's group F is quadratic. Invent. Math., in press. ArXiv math.GR/0211395.

[68] Higman, G., Subgroups of finitely presented groups. Proc. Roy. Soc. Ser. A 262 (1961), 455-475.

[69] Higman, G., A finitely generated infinite simple group, J. London Math. Soc. 26 (1951), 61-64.

[70] van Kampen, E.R., On some lemmas in the theory of groups. Amer. J. Math. 55 (1933), 268-273.

[71] Kharlampovich O.G., Myasnikov A.G., Irreducible affine varieties over a free group I and II, J. Algebra 200 (1998), 472-516 and 517-570. 
[72] Kirby, R., Problems in low-dimensional topology. AMS/IP Stud. Adv. Math., Geometric topology (Athens GA, 1993), 35-473, Amer. Math. Soc., Providence, RI, 1997.

[73] Kleiner, B.; Leeb, B.; Rigidity of quasi-isometries for symmetric spaces and Euclidean buildings. IHÉS Publ. Math. 86 (1997), 115-197.

[74] Lawson H.B., Yau S.T., Compact manifolds of nonpositive curvature. J. Differential Geom. 7 (1972), 211-228.

[75] Lubotzky, A., Tannaka duality for discrete groups. Amer. J. Math. 102 (1980), 663689.

[76] Miller, C. F., On group-theoretic decision problems and their classification. Annals of Math. Studies 68, Princeton Univ. Press, Princeton, N.J., 1971.

[77] Mosher L., Mapping class groups are automatic. Ann. of Math. 142 (1995), 303-384.

[78] Nabutovsky, A.; Weinberger, S., The fractal nature of Riem/Diff. I. Geom. Dedicata 101 (2003), 1-54.

[79] Neumann W., Reeves, L., Central extensions of word hyperbolic groups, Ann. of Math. 145 (1997), 183-192.

[80] Papasoglu, P., On the asymptotic cone of groups satisfying a quadratic isoperimetric inequality, J. Differential Geometry, 44 (1996), 789-806.

[81] Platonov V., Tavgen, O.I., Grothendieck's problem on profinite completions of groups. Soviet Math. Doklady 33 (1986), 822-825.

[82] Remeslennikov, V.N., ヨ-free groups. Sibirsk. Mat. Zh. 30 (1989), 193-197.

[83] Riley, T.R., Higher connectedness of asymptotic cones. Topology 42 (2003), 12891352 .

[84] Riley, T.R., Filling functions. ArXiv math.GR/0603059.

[85] Rips, E., Subgroups of small cancellation groups. Bull. London Math. Soc. 14 (1982), $45-47$.

[86] Sapir, M.V., Birget, J-C., Rips, E., Isoperimetric and isodiametric functions of groups, Ann. of Math. 157 (2002), 345-466.

[87] Sela, Z., Diophantine geometry over groups. I. Makanin-Razborov diagrams. IHÉS Publ. Math. 93 (2001), 31-105.

[88] Z. Sela, The isomorphism problem for hyperbolic groups I. Ann. of Math. 141 (1995), 217-283.

[89] Stallings, J.R., A finitely presented group whose 3-dimensional homology group is not finitely generated. Amer. J. Math. 85 (1963) 541-543.

[90] Short H., Groups and combings. Preprint, ENS Lyon, 1990.

[91] Vogtmann, K., Automorphisms of free groups and Outer Spaces. Geom. Dedicata 94 (2002), 1-31.

[92] Wilton H., Subgroup separability of limit groups. PhD thesis, Univ. London, 2006.

[93] Wise, D., Incoherent negatively curved groups. Proc. Amer. Math. Soc. 126 (1998), 957-964.

[94] Wise, D., A residually finite version of Rips's construction. Bull. London Math. Soc. 35 (2003), 23-29. 
Department of Mathematics

Imperial College London

London SW7 2AZ

E-mail: m.bridson@imperial.ac.uk 